\title{
¿POR QUÉ QUIERO SER PROFESOR DE EDUCACIÓN FÍSICA? ANÁLISIS DEL CAMBIO DE PERCEPCIÓN DURANTE LA FORMACIÓN UNIVERSITARIA Y LOS PRIMEROS AÑOS DE LA CARRERA DOCENTE
}

\author{
POR QUE É QUE EU QUERO SER PROFESSOR DE EDUCAÇÃO \\ FÍSICA? ANÁLISE DA MUDANÇA NA PERCEPÇÃO DURANTE A \\ FORMAÇÃO UNIVERSITÁRIA E ANOS INICIAIS DA CARREIRA \\ DOCENTE
}

\begin{abstract}
WHY DO I WANT TO BE A PHYSICAL EDUCATION TEACHER? ANALYSIS OF THE CHANGE IN PERCEPTION DURING UNIVERSITY EDUCATION AND EARLY YEARS OF TEACHING CAREER
\end{abstract}

\author{
David Hortigüela Alcalá*, Gustavo González Calvo**, \\ Ángel Pérez-Pueyo***
}

Palabras clave: Educación física. Maestros.

Rol profesional. Motivación.
Palavras chave: Educação física. Professores escolares. Papel profissional. Motivação.

Keywords: Physical education. School teachers. Professional role. Motivation.
Resumen: El presente estudio tiene dos objetivos: a) analizar la evolución de la percepción de futuros maestros EF de primer, cuarto curso de carrera y egresados; b) contrastar la realidad profesional del maestro de EF con la valoración de profesores universitarios de EF. Participaron 10 estudiantes en un diseño longitudinal de seis años, dividido en tres fases $(2012,2015$ y 2017). También participaron ocho docentes universitarios. Se utilizó un enfoque cualitativo con dos instrumentos de recogida de datos y se establecieron tres categorías de análisis. Los resultados mostraron cómo, en primer curso, los estudiantes dudan sobre qué es ser docente en EF, mientras que en cuarto curso muestran desmotivación respecto a la posibilidad de serlo. Por su parte, los egresados reflejan la incoherencia existente entre la formación universitaria recibida y la exigencia del sistema de acceso a la función pública, cuestión sobre la que los profesores universitarios no muestran concordancia.

Resumo: O presente estudo tem dois objetivos: a) analisar a evolução da percepção dos futuros professores de EF do primeiro, quarto ano de carreira e graduados; b) contrastar a realidade profissional do professor de EF com a avaliação dos professores universitários de EF. Dez alunos participaram de um projeto longitudinal de seis anos, dividido em três fases (2012, 2015 e 2017). Oito professores universitários também participaram. Foi utilizada uma abordagem qualitativa com dois instrumentos de recolha de dados e foram estabelecidas três categorias de análise. Os resultados mostraram como, no primeiro ano, os alunos duvidam do que é ser professor na EF, enquanto no quarto ano mostram falta de motivação em relação à possibilidade de se tornar um. Por outro lado, os graduados refletem a incoerência existente entre a formação universitária recebida e a exigência do sistema de acesso ao serviço público, uma questão sobre a qual os professores universitários não concordam.

Abstract: The present study has two objectives: a) to analyse the evolution of the perception of future EF teachers of first, fourth year of career and graduates; $b$ ) to contrast the professional reality of the EF teacher with the assessment of EF university professors. Ten students participated in a six-year longitudinal design, divided into three phases (2012, 2015 and 2017). Eight university professors also participated. A qualitative approach was used with two data collection instruments. The results showed how, in the first year, students doubt what it is like to be a teacher in EF, while in the fourth year they show a lack of motivation regarding the possibility of becoming one. On the other hand, the graduates reflect the incoherence existing between the university training received and the requirement of the system of access to the public service, an issue on which the university professors do not agree.
*Universidad de Burgos. Burgos, España.

E-mail:

dhortiguela@ubu.es

**Universidad de Valladolid Valladolid, España.

E-mail:

gustavo.gonzalez@uva.es

***Universidad de León. León España.

E-mail:

angel.perez.pueyo@unileon.es

Recebido em: 03-06-2019 Aprovado em: 17-02-2020 Publicado em: 08-03-2020 (c) (i) (8) Licence 


\section{INTRODUCCIÓN}

Motivación y vocación docente son dos factores que se asocian a la calidad de la enseñanza, relacionándose directamente con el tipo de formación recibida (MASSARI, 2014). Este hecho cobra especial relevancia en el área de EF, ya que el concepto de corporalidad tiene una dimensión tan amplia e interdisciplinar que debe ser concebida de nuevo y reconstruida con asiduidad (PHILPOT, 2016). Por ello, y a pesar de que la literatura científica ha dado especial relevancia al análisis de la motivación docente desde diversidad de perspectivas, no se han estudiado tanto las razones, motivos, y sobre todo la evolución de pensamiento que tienen los futuros maestros de EF para llegar a ejercer la profesión. Aunque se podría presuponer que todo estudiante que se inicia en la carrera de maestro debiera tener una cierta motivación hacia lo que, en términos generales, conlleva la profesión docente, no es siempre así. Las principales razones que a muchos les lleva a cursar esta titulación son: a) no haber sido admitidos en titulaciones con un corte de nota más elevada; b) sus experiencias previas positivas en EF y en la práctica de actividad física y deporte; y/o c) la relativa facilidad con la que prevén obtener la titulación (O'NEIL; RICHARDS, 2018). Por un lado, esta situación refleja la falta de orientación académica ofrecida desde la educación secundaria obligatoria y, por otro, la escasa valoración social hacia la función del maestro y la escuela (MCEVOY; HEIKINARO-JOHANSSON; MACPHAIL, 2017).

Ante este contexto, otro análisis de gran interés radica en la combinación de opciones laborales que giran alrededor de la formación inicial de maestros EF. Si bien los estudios están claramente orientados hacia la docencia, suele ser habitual que los estudiantes durante y después de la carrera desempeñen puestos docentes relacionados con la actividad física en cualquiera de sus vertientes (WEBSTER; NESBITT, 2017): gimnasios, clases extraescolares, monitores de ocio y tiempo libre... Las razones que justifican este hecho son variadas, yendo desde la estimulación a ganar un dinero mientras se estudia la carrera, hasta la necesidad de optar por otras opciones laborales debido a la complejidad de acceder a la función pública docente. Esta falta de definición sobre la objetividad profesional del maestro, junto otros factores estructurales, hace que en cierta medida la calidad de la formación inicial se vea mermada y debamos de reflexionar sobre cuáles son los fines y principios de la materia (NÍ CHRÓINÍN; FLETCHER; O’SULLIVAN, 2018).

En este sentido, es preciso analizar y reflexionar sobre cuáles son las motivaciones de los estudiantes hacia el desempeño profesional del maestro de EF, comprobando si estas evolucionan a lo largo de los años y las razones por las que suceden estos cambios. Además, esto permite comprobar el nivel de coherencia existente entre la formación inicial recibida y las posibilidades reales de llegar a ser docente con un pensamiento crítico (RAIKOU: KARALIS; RAVANIS, 2017). Esto se convierte en clave y fundamental si pretendemos que la EF cobre un lugar transcendente dentro del sistema educativo y aporte así a una educación de calidad integrada (SUNDARESAN; DASHOUSH; SHANGRAW, 2017).

Por ello, y con el fin de avanzar en este campo de estudio, parece necesario cuestionar de inicio la motivación intrínseca del docente. De hecho, algunas de las 
razones que los futuros docentes exponen para ser maestros se basan en aspectos extrínsecos como la estabilidad laboral y/o las largas vacaciones con las que se cuentan (JIYING; HONGBIAO, 2016).

Si no se crea una identidad profesional propia en la formación inicial del profesorado, se carecerá de un aporte intencionado al desarrollo de la materia y a la evolución de la escuela (GONZÁLEZ-CALVO; HORTIGÜELA; BARBA-MARTÍN; BORES, 2019). Esta idea, que pasa desapercibida de un modo preocupante, es de especial gravedad, ya que, si consideramos la EF como un pilar básico del bienestar social, se deberían centrar los esfuerzos institucionales y pedagógicos en estructurar con rigor tanto el acceso a las titulaciones de maestro como la utilidad de la formación adquirida (SOLTYK, 2017). En este sentido, la única posibilidad de garantizar esta construcción de identidad profesional se centra en ir más allá de los propios contenidos instrumentales de las asignaturas y centrarse en la adquisición de competencias transversales y docentes de aplicación en los centros educativos (LLEIXÀ; GONZÁLEZ-ARÉVALO; BRAZ-VIEIRA, 2016). Esto hecho, junto con la implantación de procesos que permitan a los futuros maestros dar voz a sus vivencias y experiencias personales, permitirá comprobar los cambios motivacionales acontecidos a lo largo de la carrera. Además, esta reflexión de los futuros docentes de EF conexiona directamente con su vínculo con la asignatura, valorando más cuál es su aporte educativo en el sistema educativo en el que se encuadra según los estándares curriculares definidos (HORTIGÜELA; PÉREZ-PUEYO; FERNÁNDEZ-RÍO, 2016).

Ante esto, podemos plantearnos varias cuestiones: ¿Están realmente motivados los futuros docentes de EF? ¿Qué les hace perder la motivación a lo largo de la titulación? ¿Es la formación inicial del profesorado de EF lo suficientemente real, práctica y útil? ¿Cuáles son los verdaderos alicientes que tienen los futuros maestros de EF por y para transformar la materia? Son muchos los interrogantes que surgen en torno a la motivación y a la formación del profesorado de EF y a los que la literatura no ha dado una respuesta en profundidad.

Por ello, y desde un enfoque crítico, se hace necesario cuestionarse estos aspectos, que, sin duda, inciden directamente en la calidad de la asignatura y en el poder transformador que tiene la misma a nivel social (HORTIGÜELA; FERNÁNDEZRíO; GONZÁLEZ-CALVO; PÉREZ-PUEYO, 2019). Solamente de este modo se puede repensar nuestra praxis, construyendo la identidad profesional de los futuros maestros de EF y ayudando a establecer la idiosincrasia de la materia bajo unos pilares de rigor sólidos (DAUENHAUER; KEATING; STOEPKER; KNIPE, 2019). Por lo tanto, y como hipótesis del trabajo, podríamos plantear que los futuros maestros de EF van modificando a lo largo de la carrera su orientación profesional hacia planteamientos más pedagógicos y didácticos, dejando atrás visiones más tendentes al entrenamiento y la deportivización.

Teniendo en cuenta todo lo anteriormente comentado, el presente estudio tiene dos objetivos: a) analizar la evolución de la percepción de futuros maestros EF de primer, cuarto curso de carrera y egresados; b) contrastar la realidad profesional del maestro de EF con la valoración de profesores universitarios de EF. Esto supone un aporte significativo a la temática de estudio, ya que además de comprobar la 
percepción de los futuros docentes de un modo longitudinal, se contrasta con la que tienen sus profesores universitarios.

\section{MATERIAL Y MÉTODO}

\subsection{PARTICIPANTES}

Participaron 10 estudiantes españoles (seis mujeres y cuatro hombres). Todos estudiaban la titulación de Grado en Primaria, con la Mención en EF en la Universidad de Burgos, España. Es decir, la formación para ser maestros en esta asignatura. En la primera toma de datos, año 2012, los participantes se encontraban en primer curso de carrera, teniendo una edad media de edad de 19.48 \pm 2.03 años. Estos mismos estudiantes fueron entrevistados en el año 2015 ( $4^{\circ}$ curso) y en el 2017, cuando ya eran egresados. En ese momento, siete de los 10 eran o habían sido maestros de EF. Solamente dos de ellos habían obtenido la plaza en la convocatoria del 2016, mientras que los cinco restantes habían trabajado con sustituciones periódicas en algún momento del curso académico. Los tres que no habían conseguido ejercer la profesión, seguían formándose y encadenando trabajos temporales mientras preparaban la oposición. Como dato a destacar, los 10 egresados tenían una nota media superior a 7,9 al finalizar la carrera. En el estudio, también participaron ocho docentes universitarios (cuatro hombres y cuatro mujeres). Todos ellos eran doctores, tenían más de siete años de experiencia universitaria y habían impartido docencia a estos estudiantes en la mención de EF.

\subsection{INSTRUMENTOS}

Se utilizaron dos instrumentos de recogida de datos: a) entrevistas individuales a los estudiantes en cada fase temporal; b) grupo de discusión con los profesores universitarios.

Las preguntas que constituyen cada uno de los instrumentos utilizados se han estructurado a partir de las tres categorías del estudio, obteniendo una mayor especificidad y rigor en los datos obtenidos (BOADUO, 2011).

Entrevistas individuales a los estudiantes en cada fase temporal. Se realizaron 10 entrevistas en cada fase temporal, es decir un total de 30 . Todas a los mismos participantes (Cuadro 1). Se abordaron cuestiones vinculadas a las motivaciones en torno a la profesión de maestro de EF, la utilidad de la formación recibida y el rol actual que tiene la asignatura en el aprendizaje del estudiante.

Cuadro 1 - Guion básico utilizado para las entrevistas a los estudiantes en 2012, 2015 y $2017^{*}$.

1 - ¿Por qué te gustaría ser maestro de Educación Física?

2 - ¿Cuáles son tus motivaciones principales para poder conseguirlo?

3 - ¿Cómo consideras la formación recibida respecto a las expectativas que tenías antes de cursar la carrera?

4 - ¿En qué consideras que se debería incidir más o dar mayor relevancia?

5 - ¿Cuál crees que es el principal aporte que realiza la EF al aprendizaje del estudiante?

6 - ¿Consideras que se le da el peso adecuado a nivel curricular y social? 
Grupos de discusión con los profesores universitarios. Se desarrolló en el 2017, coincidiendo con la tercera y última toma de datos de los estudiantes egresados. Se empleó un guion semiestructurado (Cuadro 2), respetando la esencia de las variables dependientes del estudio y de las entrevistas realizadas a los estudiantes. Uno de los investigadores del estudio fue el portavoz y moderador, planteando cada una de las cuestiones a todo el grupo y permitiendo una participación elevada. Esta situación permitió a los investigadores recoger la información sobre cada una de las cuestiones y posteriormente someterla a las técnicas de triangulación, depuración y saturación (OLIVER-HOYO; ALLEN, 2006). El proceso de triangulación se llevó a cabo tanto entre el contenido de cada uno de los instrumentos como entre instrumentos, buscando la información coincidente y asignándola a cada una de las categorías de análisis de los resultados. En el caso de que algún dato obtenido no atendiera a la linealidad con los objetivos del estudio, esta era eliminada.

Cuadro 2 - Guion básico utilizado para el grupo de discusión con los profesores*.

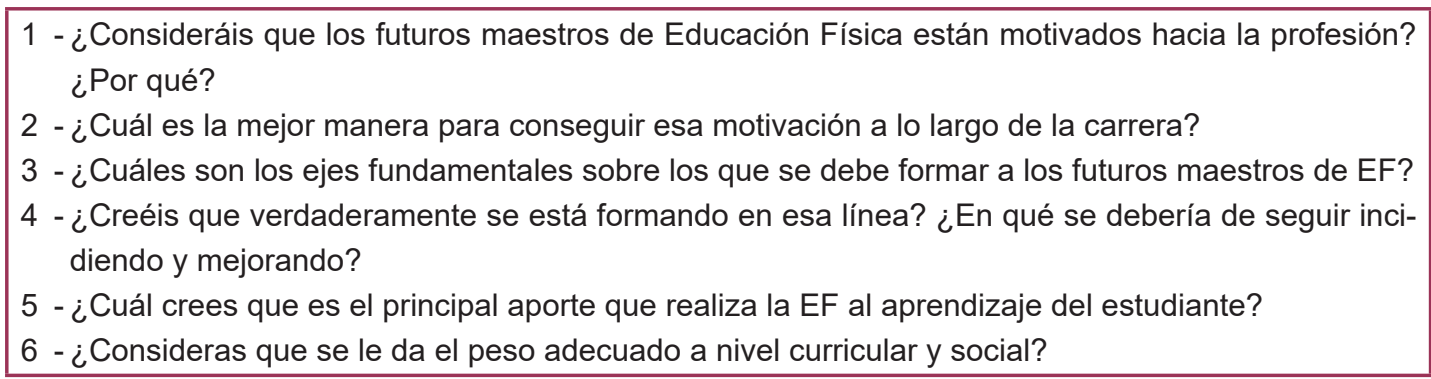

Fuente: elaboración propia

*Las preguntas 5 y 6 son idénticas a las realizadas a los estudiantes

\subsection{DISEÑO Y PROCEDIMIENTO}

Es un diseño longitudinal con tres medidas de toma de datos, con el fin de ver la evolución de los pensamientos de los mismos estudiantes a lo largo del tiempo. Este diseño fue posible llevarlo a cabo ya que uno de los investigadores impartió diversas asignaturas a los estudiantes durante la carrera, así como un curso de preparación de las oposiciones una vez que ya la habían acabado. Además, esta percepción es contrastada con la visión profesorado que les ha formado a lo largo de la titulación, algo que favorece la amplitud del espectro del tema abordado (WHITTLE; TELFORD; BENSON, 2018). La investigación se llevó a cabo en un contexto en el que la tasa de reposición pública se había abierto al 100\%, lo que implica que todas las jubilaciones existentes eran ofertadas en las convocatorias docentes. Sin embargo, las listas de interinos son extensas, lo que limita en gran parte la incorporación de aspirantes que no tienen experiencia profesional.

La investigación se ha estructurado en cuatro fases bien diferenciadas:

Fase 1. Estructuración del estudio y planificación del cronograma de intervención: cada curso escolar, uno de los investigadores tomaba datos a sus alumnos respecto a sus aprendizajes, vivencias y expectativas ante la docencia de EF. Por ello, y como impartía docencia a los estudiantes al principio y al final de la carrera, surgió la idea de realizar este estudio longitudinal. 
Fase 2. Realización de entrevistas a los estudiantes de cuarto curso: a partir de los datos tomados a los alumnos en el año 2012 y teniendo la oportunidad de impartir docencia a la mayoría de ellos en el año 2015, justo antes de finalizar la carrera, se escogieron a 10 estudiantes ya entrevistados para realizarles de nuevo la entrevista con el mismo guion. Estas se realizaron de un modo individual en horario de tutorías, teniendo una duración aproximada de 30 minutos cada una. Todas fueron grabadas en audio y transcritas para su análisis posterior. Se garantizó el anonimato de sus respuestas y la confidencialidad de los datos, destacando que sus respuestas no influirían en nada en la calificación de la asignatura/s.

Fase 3. Grupo de discusión con docentes universitarios: este grupo de discusión se realizó unas semanas después de las entrevistas a los estudiantes de cuarto curso. Estos docentes, compañeros de uno de los investigadores, fueron citados un día por la mañana en un seminario de la facultad. Tuvo una duración de 90 minutos y desde el inicio se comentó al grupo la importancia que tenían sus respuestas para la investigación. La sesión fue grabada en vídeo para una mejor recapitulación de los datos tras su visionado. La estructura del grupo de discusión se basó en la profundización de la temática del estudio con el fin de llegar a una conversación reflexiva, en un ambiente distendido y atendiendo a un criterio de proporcionalidad en las intervenciones (NGOZWANA, 2018).

Fase 4. Reanudación de contacto con estudiantes egresados y realización de entrevistas: dos años después de la realización de entrevistas con los estudiantes, se contactó con ellos vía telefónica para, además de conocer su situación personal, concretar una cita con ellos para realizar la entrevista. En seis de los casos fue posible hacerla presencial, mientras que, con otros cuatro, al encontrarse fuera de la ciudad se hizo telefónicamente, grabando la llamada.

\subsection{ANÁLISIS EMPLEADO}

Se ha empleado un enfoque cualitativo para conocer, dando voz a los participantes, cuál ha sido su evolución de pensamiento respecto a la construcción de su identidad profesional como futuros docentes de EF. Para ello, es fundamental atender a las vivencias y experiencias adquiridas a lo largo de su formación (BUARAPHAN, 2016), más si cabe cuando los investigadores han sido partícipes de la misma. Esta situación, implantada bajo un enfoque interpretativo, permite comprobar las interacciones existentes ante un mismo objeto de estudio, triangulando la información obtenida en los instrumentos de recogida de datos empleados (RICHARDS; ANDREW; HEMPHILL, 2018). Además, se aborda un componente socioeducativo de especial relevancia como la enseñanza de la EF, lo que otorga más identidad y coherencia a los enfoques cualitativos. La validez en las descripciones de las entrevistas se garantizó al ser grabadas en audio, lo que permitió que después se importaran al software de análisis sin perder nada de información. Este protocolo también se llevó a cabo con los grupos de discusión asegurando así la linealidad en 
el análisis. Además, los investigadores fueron comparando y contrastando cada una de las respuestas antes de saturar los resultados otorgando validez interpretativa a los resultados.

Con el objetivo de garantizar la confiabilidad, transferibilidad y credibilidad de los resultados, se realizó una codificación de los extractos de textos más significativos en cada uno de los instrumentos, usando para ello los patrones coincidentes cruzados (SALDAÑA, 2009). Los investigadores formaron parte de manera activa en el trabajo de campo, reflexionando a lo largo del proceso sobre la influencia de los acontecimientos. A través de la estructura del modelo de la "Grounded Theory» (STRAUSS; CORBIN, 2002), se articuló la información, agrupándola por ejes temáticos en las categorías generadas mediante a una codificación selectiva, abierta y axial.

\subsubsection{GENERACIÓN DE CATEGORÍAS Y SU CATEGORIZACIÓN}

Una vez que se transcribieron los datos de cada serie de entrevistas y del grupo de discusión, se volcaron al programa de computación y análisis WEFT QDA. A través de la saturación de textos e ideas coincidentes y el tratamiento de ejes temáticos, se agrupó la información en las tres categorías iniciales del estudio: 1) motivaciones para ser docente de EF; 2) utilidad de la formación impartida en la universidad y 3) aporte de la EF al aprendizaje del alumnado y al sistema educativo. Estas categorías, comunes a los instrumentos de recogida de datos, se encuentran en relación con el objeto de estudio, respetando así los criterios de especificidad y coherencia que debe de tener toda investigación cualitativa (TRAINOR; GRAUE, 2014).

- Motivaciones para ser docente de EF: se atiende a todas las razones y alicientes que llevan a los estudiantes querer ser docentes en esta área, analizando los patrones comunes de incentivo y las metas para llegar a serlo.

- Utilidad de la formación impartida en la universidad: se analiza en qué medida la formación metodológica y didáctica de la enseñanza de la EF es percibida cómo útil de cara al desempeño de la profesión futura de maestro.

- Aporte de la EF al aprendizaje del alumnado y al sistema educativo: se incide en el análisis de cuáles son las principales contribuciones que actualmente la EF puede hacer al aprendizaje del estudiante, teniendo presente su rol dentro del currículo y la escuela.

\subsubsection{CODIFICACIÓN DE INSTRUMENTOS DE RECOGIDA DE DATOS}

Se utilizan diferentes acrónimos para identificar los extractos de texto con el instrumento de recogida de datos del que proviene. En relación a las entrevistas a los estudiantes de la primera toma de datos, año 2012, se emplea (EE1), para las de la segunda toma de datos, año 2015, se utiliza (EE2), y para las de la tercera con egresados, año 2017, (EE3). Respecto al grupo de discusión con docentes, se utiliza (GDD). 


\title{
3 RESULTADOS
}

Toda la información extraída de los instrumentos de recogida de datos se agrupa en las tres categorías del estudio. Mediante el análisis de patrones cruzados se presentan el número de extractos literales de texto resultantes, mostrándose aquellos más significativos y coincidentes. 3.1 MOTIVACIONES PARA SER DOCENTE DE EF: (321 EXTRACTOS DE TEXTO).

Se observa cómo los estudiantes en primer curso no tienen demasiado claro por qué quieren ser docentes de EF y eligieron la titulación:

\begin{abstract}
Sinceramente, me gustaría ser profesor de EF ya que soy deportista y que mejor que enseñarlo en clase y dedicarme a ello [...]. Pues mi idea inicial era hacer INEF, pero al no darme la nota entré en magisterio [...]. Supongo que me gustará también trabajar con los más pequeños o dar clases de deporte". "El deporte para mí es una de las cosas más importantes en la vida, y poder dedicarme a ello es una motivación. Mi padre y mi madre son profesores y siempre me han insistido mucho en que yo le sea $[\ldots]$. (EE1).
\end{abstract}

Esta percepción ya ha cambiado en gran parte cuando los alumnos están en cuarto curso, teniendo más claras las razones y motivos de llegar a ser docentes de EF:

\begin{abstract}
Sinceramente, tengo mucho más claro que nunca que quiero ser maestro de EF [...]. Tras cursar las asignaturas de la mención, y didáctica claro, me he dado cuenta que el cuerpo puede trabajarse desde diferentes dimensiones y enfoques [...]". "Tenemos mucho que hacer y aportar en este ámbito". "Mi enfoque inicial era más deportivo, ahora hemos visto los aspectos motivacionales y de relación social sobre el cuerpo son los más importantes". "Ufff, es una pasada echar la vista atrás y ver cómo ha cambiado mi manera de ver las cosas [...]. Ahora tengo claro que la EF ha de ser inclusiva y llegar a todos los alumnos (EE2).
\end{abstract}

Por su parte, los estudiantes egresados muestran una desmotivación respecto a la posibilidad de ser maestros de EF, debido a la complejidad del acceso a la función pública. Los docentes universitarios destacan la gran disparidad de motivación entre los estudiantes:

La motivación es alta durante la carrera, pero cuando te encuentras con la realidad es muy complejo poder trabajar". "En dos años he trabajado tres meses, y no será porque no me he formado y estudiado [...]". "Da mucha rabia ver que puedes contribuir mucho a la asignatura pero que el sistema no te deja" "Sinceramente, me han dado ganas de tirar la toalla en varias ocasiones y no opositar más. (EE3).

La motivación de los estudiantes es muy dispar entre ellos, y a lo largo de los cursos". "La experiencia nos dice que el que trabaja y es constante acaba trabajando de maestro". "Hay estudiantes que desde el primer día ves que quieren transformar la materia en los prácticum y otros que, en fin". "Hay que reconocer que el enfoque y tratamiento que se les da algunas asignaturas no ayuda a transmitir esta motivación (GDD).

\subsection{UTILIDAD DE LA FORMACIÓN IMPARTIDA EN LA UNIVERSIDAD: (305 EXTRACTOS DE TEXTO).}

Los estudiantes de primer curso valoran la formación recibida hasta el momento como demasiado teórica, incidiendo en la necesidad de recibir más ejemplos de aula: 
Tras este primer curso, estoy un poco decepcionada la verdad, hay demasiadas teorías, clasificaciones [...]". "Te pasas la mayoría del tiempo imaginando lo que será un centro educativo y no eres capaz de imaginar si eso que te están contando se podrá utilizar en clase [...]. "Espero que esto cambie, si no, me replanteo dejar la carrera". "Las asignaturas de EF son mucho más motivantes que las otras, el clima es muchísimo mejor en clase $[\ldots]($ EE1).

En relación a los estudiantes de $4^{\circ}$ curso, se observa cómo su percepción es muy dispar entre asignaturas y docentes. Valoran positivamente cuando se dan ejemplos de aula concretos actuales en relación a la EF:

No hay color entre unos docentes y otros [...]. Algunos te dan ejemplos reales de las clases de EF, lo que te hace ponerte de verdad en lugar de un maestro [...]". "Algunos profesores parece que no han visto un colegio en los últimos 30 años, incluso te hablan de legislación que ya no está vigente o de cosas que están muy desfasadas". "Hay con algún profesor con el que no he aprendido absolutamente nada [...]. Algunos nos piden implicación, pero luego ellos ni contestan al email". "Con alguna de la formación recibida, la verdad es que se te cae el alma a los pies y desmotiva para ser docente de EF (EE2).

Algunos de los egresados critican la poca formación que han recibido a lo largo de la carrera respecto a la oposición. También se destaca el vacío que sienten al finalizar la carrera. Por su parte, los docentes universitarios presentan disparidad respecto a cuáles deben de ser los fines de la formación:

Lo que no me parece lógico es que salvo un profesor nadie nos haya hablado de las oposiciones de verdad [...]. Y muchas veces cuando se hace es de manera abstracta y sin concretarnos nada". "Es fundamental saber a qué te vas a enfrentar al acabar y la verdad es que la realidad te pega luego un viaje fuerte, en cierta manera te sientes olvidado". "Es triste, pero he aprendido más en un año de academia que en gran parte de la carrera [...]". (EE3).

"No creo que debamos de formarles en relación a la oposición ni mucho menos [...]. El enfoque ha de ser global, que tengan ideas pero que ellos decidan el tipo de docentes de EF que quieren ser y lo que quieren aportar". "Pues yo considero que sí que es necesario prepararles para lo que se van a encontrar fuera, esto para nada está reñido con darles recursos prácticos que puedan implantar en sus aulas" (GDD).

\subsection{APORTE DE LA EF AL APRENDIZAJE DEL ALUMNADO Y AL SISTEMA EDUCATIVO (347 EXTRACTOS DE TEXTO).}

El enfoque que dan los estudiantes de primer curso a la EF se dirige sobre todo a la salud y la prevención de obesidad, destacando la importancia de la realización de práctica deportiva por encima de aspectos más integradores:

\footnotetext{
Hay mucha obesidad en la actualidad, y este es un problema que podemos revertir desde la EF [...]. Es importante hacer deporte, y cuanto más hagan mejor, para eso están las clases de EF. Si queremos que los niños dejen de ser sedentarios, tenemos que dar más caña en las clases de EF. Recuerdo cuando estaba en el instituto y había chicas que no se movían en clase y encima aprobaban, yo quiero cambiar eso (EE1).
}

Se observa cómo los estudiantes de cuarto curso ya tienen más claro el aporte de la asignatura, debido en parte a la formación recibida:

La EF aporta muchísimo, más que cualquier otra asignatura [...]. Ya pude ver en el prácticum cómo los niños la disfrutan, y tuve la oportunidad de poner cosas en práctica que nos enseñaron en la carrera, todo un lujo 
y espero poder ser maestro en poco tiempo". "He disfrutado mucho impartiendo docencia de EF, tenemos tantas posibilidades con los alumnos [...] "Tengo claro que para aportar en los colegios la EF tiene que utilizarse globalmente y en relación con otras asignaturas" (EE2).

De nuevo la percepción de los egresados es algo desmotivante. Muestran una desconexión entre la formación recibida y lo vivenciado en los centros. Entre los docentes universitarios chocan los discursos de participación y rendimiento:

Cuando acabas la carrera tienes muy claro cuál ha de ser el enfoque de la EF, pero cuando llegas a un centro ves que las cosas no siempre son así. Hay muchos profes que pasan de la EF y el claustro no suele darla mucha importancia. Es difícil luchar por otro modelo de EF cuando en los centros te encuentras con enfoques puramente tradicionales. A veces los alumnos hay que cambiar las experiencias que tienen los niños previamente, porque no todo es jugar y tiempo libre (EE3).

Me preocupa que con el movimiento tan fuerte de la EF integral se pierda el fin principal de la asignatura, la búsqueda de salud, el trabajo intencionado de la motricidad, el esfuerzo [...].La EF en primaria aislada de otras asignaturas y contenidos pierde su total sentido, no podemos aislar la motricidad y la salud de un enfoque educativo e integrado (GDD)

\section{DISCUSIONES}

Los dos objetivos de la investigación fueron: a) analizar la evolución de la percepción de futuros maestros EF de primer, cuarto curso de carrera y egresados; b) contrastar la realidad profesional del maestro de EF con la valoración de profesores universitarios de EF. Respecto al primer objetivo, se ha comprobado cómo los estudiantes a lo largo de los cursos van teniendo más claro por qué quieren ser maestros de EF, demandando una formación más práctica y real sobre cómo impartirla en los centros educativos. Por su parte, los egresados manifiestan un vacío al finalizar la carrera respecto a cómo preparar el proceso de oposición, mientras que entre los docentes universitarios surgen contradicciones sobre los fines de la enseñanza en la formación inicial.

En relación a la primera categoría de análisis relativa a las motivaciones para ser docente de EF, se ha observado la disparidad de opiniones sobre por qué quieren ser docentes de EF. Esta situación arroja una reflexión crítica importante, ya que, si tan relevante es la EF, la vocación por llegar a ser docente y el prestigio de la carrera debiera de ser mucho más elevado, dejando claro desde el inicio la relación entre teoría y práctica de los programas de estudio (SPITTLE; SPITTLE, 2016). Soltyk (2017) indica cómo la formación inicial del profesorado de EF es el eje fundamental para mejorar la sociedad del futuro a nivel internacional y eso solamente se consigue si desde el comienzo existe una predisposición alta hacia el aprendizaje de los maestros, apoyado por una sólida estructura académica. Sin embargo, esta situación cambia claramente en los estudiantes de cuarto curso, teniendo un enfoque más dirigido al plano social y motivacional que al plenamente deportivo. Estos datos son curiosos y contrastan con algunas investigaciones como la de Richards, Housner y Templin (2018) que demuestran que en la formación de los docentes de EF siguen predominando las metodologías tradicionales y los contenidos deportivos, siendo necesario reconducir los estudios hacia el concepto de socialización. Otro resultado 
de interés en esta categoría ha sido la desmotivación presentada por los egresados respecto a la posibilidad de ser docentes en el sistema público. En este sentido, Cardina y James (2018) abogan por establecer una conexión entre las habilidades necesarias de los docentes de EF y la demanda en los centros educativos, estableciendo así una coherencia en las oportunidades de desarrollo profesional.

Respecto a la segunda categoría de estudio relativa a la utilidad de la formación impartida en la universidad, que los estudiantes de primer curso la perciban como demasiado teórica. Esta situación, demasiado habitual y recurrente, parece necesario que sea analizada desde un prisma más general. Philpot (2016) aboga por emplear enfoques basados en la pedagogía crítica, con el fin de que los docentes reflexionen profundamente sobre la practicidad implícita de toda formación. Esta disrupción entre teoría y práctica parece ser superada por los estudiantes de cuarto, manifestando la disparidad de implicación que existe entre unos docentes y otros. Estudios como el de Visser-Wijnveen, Stes y Van Petegem (2014) reflejan la disparidad existente entre las motivaciones del docente universitario hacia la enseñanza, señalando los factores de efectividad personal percibida, el interés y el esfuerzo hacia la docencia como los más destacados. Además, dentro de los docentes más motivados, predominó el factor de priorización del proceso de enseñanza sobre el contenido. Este pensamiento de disparidad entre profesores es coincidente en los egresados, manifestando que la formación recibida debería de estar más relacionada con lo que se te exige en el mercado laboral. Ante esto, los docentes universitarios difieren entre ellos. Robinson (2018) destaca que uno de los problemas en la docencia universitaria es la falta de coordinación entre los distintos departamentos, lo que hace que no se tengan claros los fines de las titulaciones. Para ello, plantea la proposición de programas basados en estrategias de aula para fomentar el interés de los estudiantes.

En la tercera categoría relacionada con el aporte de la EF al aprendizaje del alumnado y al sistema educativo, la visión de los estudiantes de primer curso se dirige fundamentalmente a la salud, a la prevención de obesidad y al deporte. Esto, sin duda, refleja cual ha sido el tipo de EF que recibieron en su formación obligatoria. Ante esta situación, y con el fin de resignificar el tratamiento de la materia, Johnson (2012) establece algunos principios fundamentales para los futuros maestros de EF como; el desafío a la conceptualización tradicional de la materia, la implicación activa en sus centros educativos y la proposición de recursos y vivencias a los estudiantes para establecer así una cultura de valor. Sin embargo, los estudiantes de cuarto curso han tenido una percepción hacia la EF más global, abordándola desde unos fines más sociales y atendiendo a los aspectos motivacionales. Es curioso que este enfoque, coincidente con los egresados desde una visión práctica de aplicación en los centros educativos, no sea tan manifiesto y aclaratorio entre los docentes universitarios. En este sentido, y partiendo de la idea de lo positivo que es reflexionar sobre paradigmas educativos diferentes, cabe pensar hasta qué punto lo es cuando se cuestionan los fines esenciales de la materia. Mcevoy, Heikinaro-Johansson y Macphail (2017) en un estudio realizado con docentes de futuros maestros de siete países muestran cómo los principales objetivos de la asignatura se centran en la máxima realización de actividad física. Además, se observa cómo sus percepciones han cambiado a lo largo del tiempo, debido a factores como los resultados de sus investigaciones, las 
expectativas sociales y la necesidad percibida de legitimar la educación física en contextos escolares y universitarios.

\section{CONCLUSIONES}

Atendiendo a los objetivos del estudio y a las categorías de análisis, podemos establecer las siguientes conclusiones: a) los estudiantes de primer curso no tiene claro por qué quieren ser docentes de EF, cambiando esta situación a lo largo de la carrera por el valor que otorgan a lo corporal en toda su dimensión; b) los estudiantes y egresados demandan una formación más práctica y actual, una mayor implicación de algún profesor y una formación y asesoramiento en relación a las oposiciones; c) los estudiantes de primer curso tienen una visión de la EF más basada en la prevención de la obesidad y la deportivización, mientras que la de cuarto y egresados va hacia enfoques más sociales. Sin embargo, los docentes universitarios presentan contradicciones sobre cuál debe de ser el tipo de formación a impartir.

El principal aporte de esta investigación ha sido mostrar cómo evoluciona la percepción de futuros maestros de EF a lo largo de su formación académica y cuando son egresados, aportando además la visión de sus profesores universitarios. Esto cubre un vacío de la literatura, al no existir investigaciones previas que aborden este ámbito de manera tan longitudinal. Sin embargo, el estudio presenta algunas limitaciones y posibles líneas futuras de trabajo. En primer lugar, podrían contrastarse estas percepciones con las de profesores de secundaria, comprobando así cuáles son las diferencias formativas entre etapas. En segundo lugar, se podrían utilizar instrumentos de registro de datos más narrativos como por ejemplo diarios reflexivos. Del mismo modo, podrían realizarse estudios de carácter longitudinal que fueran aplicados a lo largo de la carrera docente o etapas de desarrollo profesional, aumentando también el número de entrevistados.

\section{REFERENCIAS}

BOADUO, Nana Adu-Pipim. Systematic Analysis and Interpretation of Collected Data for a Research Study: A Practical Methodological Framework for Writing Research Report. Educational Research and Reviews, v.6, n 2, p. 140-146, 2011.

BUARAPHAN, Khajornsak. The Development of Qualitative Classroom Action Research Workshop for In-Service Science Teachers. Asia-Pacific Forum on Science Learning and Teaching, v. 17, n.1, p. 1-12, 2016.

DAUENHAUER, Brian; KEATING, Xiaofen; STOEPKER, Peter; KNIPE, Robert. State Physical Education Policy Changes from 2001 to 2016. Journal of School Health, v.89, n.6, p. 485-493, 2019.

GONZÁLEZ-CALVO, Gustavo; HORTIGÜELA, David; BARBA-MARTíN, Raúl; BORES, Daniel. La foto-elicitación como instrumento para profundizar en la construcción de las subjetividades corporales de maestros de educación física en formación. Movimento, v. 24, n. 4 , p. 1249-1264, 2019. 
HORTIGÜELA, David; PÉREZ-PUEYO, Ángel; FERNÁNDEZ-RÍO, Javier. Entender y enseñar la educación física en la formación inicial del profesorado: estudio de caso en España y Costa Rica. Movimento, v.22, n. 4, p. 1049-1062, 2016.

HORTIGÜELA, David; FERNÁNDEZ-RÍO, Javier; GONZÁLEZ-CALVO, Gustavo; PÉREZPUEYO, Ángel. Comparing effects of a TPSR training program on prospective physical education teachers' social goals, discipline and autonomy strategies in Spain, Chile and Costa Rica. Physical Education and Sport Pedagogy, v. 24, n.3, p. 220-232, 2019.

JIYING, Han; HONGBIAO, Yin. Teacher Motivation: Definition, Research Development and Implications for Teachers. Cogent Education, v. 3, n. 1, p. 12-17, 2016.

JOHNSON, Tyler. G. (2012). The Significance of Physical Education Content: "Sending the Message" in Physical Education Teacher Education. Quest, v. 63, n. 3, p. 187-196, 2012.

LLEIXÀ, Teresa; GONZALEZ-AREVALO, Carles; BRAZ-VIEIRA, Marcelo. Integrating Key Competences in School Physical Education Programmes. European Physical Education Review, v. 22, n. 4, p. 506-525, 2016.

MASSARI, Gianina-Ana. Motivation for Teaching Career of Students from Early Childhood Education and Primary School Pedagogy. Acta Didactica Napocensia, v. 7, n. 4, p. 1-6, 2014.

MCEVOY, Eileen; HEIKINARO-JOHANSSON, Pilvikki; MACPHAIL, Ann. Physical Education Teacher Educators' Views Regarding the Purpose(s) of School Physical Education. Sport, Education and Society, v. 22, n. 7, p. 812-824, 2017.

NÍ CHRÓINÍN, Déirdre; FLETCHER, Tim; O'SULLIVAN, Mary. Pedagogical Principles of Learning to Teach Meaningful Physical Education. Physical Education and Sport Pedagogy, v. 23, n. 2, p. 117-133, 2018.

NONGZWANA, Nomazulu. Ethical Dilemmas in Qualitative Research Methodology: Researcher's Reflections. International Journal of Educational Methodology, v.4, n. 1, p. 19-28, 2018.

OLIVER-HOYO, Maria; ALLEN, DeeDee. The Use of Triangulation Methods in Qualitative Educational Research. Journal of College Science Teaching, v. 35, n. 4, p. 42-47, 2006.

O'NEIL, Kason; RICHARDS, K. Andrew R. Breaking from Traditionalism: Strategies for the Recruitment of Physical Education Teachers. Journal of Physical Education, Recreation \& Dance, v. 89, n. 2, p. 34-41, 2018.

PHILPOT, Rod. Physical Education Initial Teacher Educators' Expressions of Critical Pedagogy(ies): Coherency, Complexity or Confusion? European Physical Education Review, v. 22, n. 2, p. 260-275, 2016.

RAIKOU, Natassa; KARALIS, Thanassis; RAVANIS, Konstantinos. Implementación de un método innovador para desarrollar ha Implementing an Innovative Method to Develop Critical Thinking Skills in Student Teachers. Acta Didactica Napocensia, v. 10, n. 2, p. 2130, 2017.

RICHARDS, K. Andrew R.; HOUSNER, Lynn D.; TEMPLIN, Thomas J. Addressing Physical Education Teacher Socialization through Standards-Based Reform of Physical Education Teacher Education. Quest, v. 70, n. 3, p. 334-353, 2018.

RICHARDS, K. Andrew R.; HEMPHILL, Michael A. A Practical Guide to Collaborative Qualitative Data Analysis. Journal of Teaching in Physical Education, v. 37, n. 2, p. 225231, 2018. 
ROBINSON, Matt. Transforming Teachers, Departments and Schools: Brain-Based Program Breaks Boundaries and Overcomes Limitations. New England Journal of Higher Education, n. 1, p. 1-12, Jan. 2018.

SALDAÑA, Johnny. The Coding Manual for Qualitative Researchers. Thousand Oaks: Sage, 2009.

SOLTYK, Oleksandr. The Peculiarities of Physical Education Teachers' Professional Training: Foreign Experience. Comparative Professional Pedagogy, v. 7, n.4, p. 39-44, 2017.

SPITTLE, Michael; SPITTLE, Sharna. Content of Curriculum in Physical Education Teacher Education: Expectations of Undergraduate Physical Education Students. Asia-Pacific Journal of Teacher Education, v. 44. n. 3, p. 257-273, 2016.

STRAUSS, Anselm L.; CORBIN, Juliet. Bases de la investigación cualitativa: Técnicas y procedimientos para desarrollar la teoría fundamentada. Medellin: Universidad de Antioquia, 2002.

SUNDARESAN, Nirup; DASHOUSH, Nermeen; SHANGRAW, Rebecca. Now That We're "Well Rounded," Let's Commit to Quality Physical Education Assessment. Journal of Physical Education, Recreation \& Dance, v. 88, n. 8, p. 35-38, 2017.

TRAINOR, Audrey A.; GRAUE, Elizabeth. Evaluating Rigor in Qualitative Methodology and Research Dissemination. Remedial and Special Education, v. 35, n. 5, p. 267-274, 2014.

VISSER-WIJNVEEN, Gerda J.; STES, Ann; VAN PETEGEM, Peter. Clustering Teachers' Motivations for Teaching. Teaching in Higher Education, v. 19, n. 6, p. 644-656, 2014.

WEBSTER, Collin A.; NESBITT, Danielle. Expanded Roles of Physical Education Teachers within a CSPAP and Implications for PETE. Journal of Physical Education, Recreation \& Dance, v. 88, n. 3, p. 22-28, 2017.

WHITTLE, Rachael J.; TELFORD, Amanda; BENSON, Amanda C. Teachers' Perceptions of How They Influence Student Academic Performance in VCE Physical Education. Australian Journal of Teacher Education, v. 43, n. 2, p. 1-25. 2018. 